\title{
Transverse instability of a bunched beam with space charge and wakefield
}

\author{
V. Balbekov* \\ Fermi National Accelerator Laboratory, P.O. Box 500, Batavia, Illinois 60510, USA
}

(Received 19 May 2011; published 19 September 2011)

\begin{abstract}
Transverse instability of a bunch in a ring accelerator is considered with space charge and wakefield taken into account. It is assumed that space charge tune shift significantly exceeds the synchrotron tune. Bunch spectrum, instability growth rate, and effects of chromaticity are studied with different bunch and wake forms. Fast instability caused by coupling of transverse modes is studied in detail. It is shown that, for monotonic wakes, the transverse mode coupling instability is possible only with a certain sign of the wake. Its threshold and growth rate are calculated precisely over a wide range of parameters.
\end{abstract}

DOI: 10.1103/PhysRevSTAB.14.094401

PACS numbers: 29.27.Bd

\section{INTRODUCTION}

Transverse instability of a bunched beam in a ring accelerator has been considered first by Pellegrini [1] and Sands [2] with synchrotron oscillations taken into account ("head-tail instability"). Coherent oscillations of a bunch were presented as a sum of uncoupled modes $\propto \exp (\operatorname{im} \phi)$ where $\phi$ is synchrotron phase. After that, Sacherer investigated the effect in depth including the so-called radial modes, which describe dependence of coherent displacement on synchrotron amplitude [3].

The role of space charge has been studied first in Ref. [4] with the assumption that corresponding tune shift is small in comparison with synchrotron tune. It was shown that the appearing tune spread causes Landau damping which suppresses most of the head-tail modes. The lowest (rigid) mode is the only universal exception from the rule, and 1-2 next modes can be unstable additionally, in dependence on the bunch shape. The results were confirmed later in Ref. [5] by an analysis of several simple models. A more detailed investigation has shown that the damping vanishes at larger tune shift [6]. Although the thresholds were not defined exactly, there was shown that the lower modes are earlier becoming the decay free.

The space charge effects at low synchrotron frequency were thoroughly investigated in recent papers [6,7]. Although very similar equations have been presented by the authors, they have arrived at the rather different conclusions.

According to Ref. [6], all the eigentunes of the bunch should obtain an imaginary part in response to a wakefield of any nature and strength. However, no concrete wakes were investigated in the work.

\footnotetext{
*balbekov@fnal.gov

Published by the American Physical Society under the terms of the Creative Commons Attribution 3.0 License. Further distribution of this work must maintain attribution to the author(s) and the published article's title, journal citation, and DOI.
}

In contrast to this, solutions with several specific wakes were presented in Ref. [7]. The conclusion was that, at zero chromaticity, small wake cannot violate bunch stability, and all its eigentunes remain to be real numbers. Wake strength should overcome a certain threshold to excite transverse mode coupling instability through approach, and coalescence of the eigentunes (vanished TMCI, by the author).

The contradiction is resolved in this paper. It is shown that some small parts of the basic equation were lost at limiting transition to zero frequency in Ref. [7]. With the parts recovered, all the eigentunes prove to be complex numbers almost in all the cases, with rare exclusions only due to special combinations of parameters. The imaginary parts do increase when the TMCI arises; however, recalculated in Ref. [6] the TMCI thresholds appreciably differ from those presented in Ref. [7].

This concern is discussed in subsequent sections. One of the base assumptions is that nonlinearity of the external field is negligible in comparison with space charge contribution. Another important assumption is that synchrotron oscillations are linear. However, the effect of chromaticity on the instability growth rate is studied and treated in the paper.

\section{BUNCH MODES EQUATION}

Wakefield of a beam produces transverse Lorenz force which can be written in the form (per unit charge)

$$
\vec{G}^{(L)}\left(t, \theta^{(L)}\right)=-\int_{0}^{t} W\left(t-t^{\prime}\right) \vec{D}^{(L)}\left(t^{\prime}, \theta^{(L)}\right) d t^{\prime},
$$

where $D^{(L)}\left(t, \theta^{(L)}\right)$ is linear density of the beam dipole moment in azimuth $\theta^{(L)}$. This usual equation refers to the laboratory frame, although the corresponding rest frame is more convenient for our purpose. Therefore the following variables and definitions will be used hereafter:

$$
\theta=\theta^{(L)}-\Omega_{0} t, \quad G(t, \theta)=G^{(L)}\left(t, \theta^{(L)}\right), \quad \text { etc. }
$$

where $\Omega_{0}$ is the bunch angular velocity. Then Eq. (1) transforms to 


$$
\vec{G}(t, \theta)=-\int_{0}^{t} W\left(t-t^{\prime}\right) \vec{D}\left[t^{\prime}, \theta+\Omega_{0}\left(t-t^{\prime}\right)\right] d t^{\prime} .
$$

With this force and space charge field taken into account, the equation of betatron oscillations of a particle in a linear external field is

$$
\frac{d^{2} x}{d t^{2}}+\Omega^{2} Q^{2} x=\frac{e E_{x}[\theta, x-\bar{X}(t, \theta), y]}{m \gamma^{3}}+\frac{e G_{x}(t, \theta)}{m \gamma},
$$

where $\Omega$ and $Q$ are the particle angular velocity and tune, and $\bar{X}(t, \theta)$ is the beam transverse displacement at azimuth $\theta(\theta=0$ in the bunch center). The space charge electric field $E_{x}$ is, possibly, a nonlinear function of transverse coordinates $x$ and $y$. Note that $d / d t$ is the total derivative in time involving longitudinal motion.

An averaging of Eq. (4) over transverse phase space gives an equation for the function $X(t, \theta, p)$ which is transverse displacement of the bunch in the point $(\theta, p)$ of longitudinal phase space [6]:

$$
\frac{\partial X}{\partial t}+\Omega_{s} \frac{\partial X}{\partial \phi}+i \Omega Q X \simeq i \Omega \Delta Q(X-\bar{X})+\frac{i e G_{x}(t, \theta)}{2 m \gamma \Omega_{0} Q_{0}} .
$$

Here $\phi$ and $\Omega_{s}=\Omega_{0} Q_{s}$ are phase and frequency of the linear synchrotron oscillations. The functions $X(t, \theta, p)$ and $\bar{X}(t, \theta)$ are related by the equation

$$
\rho(\theta) \bar{X}(t, \theta)=\int_{-\infty}^{\infty} F(\theta, u) X(t, \theta, p) d p,
$$

where $\rho(\theta)$ is linear bunch density associated with longitudinal distribution function $F$ :

$$
\rho(\theta)=\int_{-\infty}^{\infty} F(\theta, p) d p .
$$

The effective space charge tune shift does not depend on transverse coordinates in Eq. (5), demonstrating that nonlinearity of the beam field does not affect the coherent oscillations $[6,8]$ :

$$
\Delta Q(\theta)=\frac{e}{2 m \gamma^{3} \Omega_{0}^{2} Q_{0}} \int_{-\infty}^{\infty} \frac{\partial E_{x}}{\partial x}(\theta, x, y) \rho_{\perp}(x, y) d x d y
$$

where $\rho_{\perp}$ is normalized steady state transverse density of the beam which just produces the electric field $\vec{E}$.

Laplace transformation in time will be applied as a next step to obtain a new variable defined by the relation

$$
Y(\theta, p)=\exp (i \chi \theta) \int_{0}^{\infty} X(t, \theta, p) \exp (i \omega t) d t
$$

where

$$
\chi=\frac{d(\Omega Q)}{d \Omega}=Q_{0}+\Omega_{0} \frac{d Q / d p}{d \Omega / d p}=Q_{0}+\zeta
$$

( $\zeta=-\xi / \eta$ is normalized chromaticity). The exponential factor is added before the integral to exclude dependence of the parameters $\Omega$ and $Q$ on momentum in Eq. (5). With zero initial conditions, the equation transforms to

$$
\begin{aligned}
(\omega & \left.-\Omega_{0} Q_{0}\right) Y+i \Omega_{0} Q_{s} \frac{\partial Y}{\partial \phi}+\Omega_{0} \Delta Q(\theta)(Y-\bar{Y}) \\
& =\frac{e \exp (i \chi \theta)}{2 m \gamma \Omega_{0} Q_{0}} \int_{0}^{\infty} W\left(t^{\prime}\right) D_{\omega}\left(\theta+\Omega_{0} t^{\prime}\right) \exp \left(i \omega t^{\prime}\right) d t^{\prime},
\end{aligned}
$$

where

$$
D_{\omega}(\theta)=\int_{0}^{\infty} D_{x}(t, \theta) \exp (i \omega t) d t .
$$

Note that a relation like Eq. (6) is valid for the functions $Y(\theta, p)$ and $\bar{Y}(\theta)$ as well.

Variable $\theta$ has a range $[-\pi, \pi]$ (one turn) whereas the actual bunch has a smaller range $\left[-\theta_{0}, \theta_{0}\right]$. Therefore, it is more convenient to use the normalized value

$$
\tau=\frac{\theta}{\theta_{0}}
$$

with a range $[-1,1]$ (it was denoted as $\theta$ in Ref. [6], but the symbol is occupied now). Then Eqs. (6) and (7) hold true with formal replacement $\tau$ instead of $\theta$, because normalization of the distribution function was not specified yet. Further, we will apply the condition

$$
\int_{-1}^{1} \rho(\tau) d \tau=1
$$

Then the linear density of the dipole moment is

$$
D_{\omega}=\frac{e \bar{X}_{\omega}}{R} \frac{d N}{d \theta}=\frac{e N \bar{Y}(\tau) \rho(\tau)}{R \theta_{0}} \exp \left(-i \chi \theta_{0} \tau\right),
$$

where $R$ is the machine radius, and $N$ is the number of particles in the bunch. Designating

$$
\frac{e^{2} N W(t)}{2 m \gamma R \Omega_{0}^{2} Q_{0}}=2 \Omega_{0} q\left(\Omega_{0} t\right),
$$

one can rewrite Eq. (11) in the form

$$
\begin{aligned}
& \left(\frac{\omega}{\Omega_{0}}-Q_{0}\right) Y+i Q_{s} \frac{\partial Y}{\partial \phi}+\Delta Q(\theta)(Y-\bar{Y}) \\
& \quad=2 \int_{0}^{\infty} q\left(\theta_{0} \tau^{\prime}\right) \exp \left[i \theta_{0} \tau^{\prime}\left(\frac{\omega}{\Omega_{0}}-\chi\right)\right] \bar{Y}\left(\tau^{\prime}+\tau\right) \rho\left(\tau^{\prime}+\tau\right) d \tau^{\prime} .
\end{aligned}
$$

Without the right-hand part, the equation has a universal solution (rigid mode):

$$
Y=\bar{Y}=1, \quad \frac{\omega}{\Omega_{0}}=Q_{0} .
$$

It is easy to add here a contribution of a reasonably weak wakefield. In the simplest case (constant wake, low chromaticity, boxcar bunch), the tune shift is

$$
\frac{\Delta \omega}{\Omega_{0}} \simeq q\left(1-\frac{2 i \theta_{0} \zeta}{3}\right)
$$

Hence, in order of value, $q$ coincides with the addition to the lowest eigentune. Note that $q$ is negative in many practical cases (e.g. resistive wall wake). According to 
Eq. (19), similar wakes should decrease tune of the rigid mode and excite its instability at $\zeta>0$. It is important that the statements hold true independently on $\Delta Q$.

\section{LOW SYNCHROTRON FREQUENCY LIMIT}

The ultimate case $Q_{s} \ll \Delta Q$ will be considered in the following sections. Then, using the methods developed in Ref. [6], one can obtain the following equation for the function $\bar{Y}(\tau)$ :

$$
\begin{aligned}
U^{2} \frac{d^{2} \bar{Y}}{d \tau^{2}}-\left(\tau+\frac{U^{2}}{\nu \rho_{c}+\rho} \frac{d \rho}{d \tau}\right) \frac{d \bar{Y}}{d \tau}+\frac{\nu\left(\nu \rho_{c}+\rho\right)}{\mu^{2} \rho_{c}} \bar{Y} \\
=\frac{2 \Delta Q_{c} \rho(\tau)}{Q_{s}^{2} \rho_{c}} \int_{0}^{\infty} q\left(\theta_{0} \tau^{\prime}\right) \exp \left[i \theta_{0} \tau^{\prime}\left(\nu \Delta Q_{c}-\zeta\right)\right] \\
\quad \times \bar{Y}\left(\tau^{\prime}+\tau\right) \rho\left(\tau^{\prime}+\tau\right) d \tau^{\prime}
\end{aligned}
$$

with

$$
\mu=\frac{Q_{s}}{\Delta Q_{c}}, \quad \nu=\frac{\omega-\Omega_{0} Q_{0}}{\Omega_{0} \Delta Q_{c}}, \quad U^{2}=\frac{1}{\rho(\tau)} \int F(\tau, u) u^{2} d u .
$$

Here and subsequently, the subindex $c$ marks the bunch center, $u$ is reduced particle momentum in the rest frame: $u^{2}=A^{2}-\tau^{2}$, where $A=\tau_{\max }$ is amplitude of synchrotron oscillation. Note that all of the solutions are regular functions, that is Landau damping plays no part in these conditions [6].

Equation (20) without the wakefield is developed in Ref. [6] where details of the derivation can be found as well. With an additional assumption $\nu \rightarrow 0$, the equation coincides with Eq. (60) of Ref. [7]. Such a limiting transition is quite legitimate in the left-hand side of Eq. (20) which, with real $\nu$, contains only real coefficients. However, it totally annihilates the imaginary part of the right-hand side at $\zeta=0$. Such was indeed the case of Ref. [7] when the conclusion was made that, at zero chromaticity, all eigentunes are real numbers as long as the wake reaches the TMCI threshold. However, it is seen now that the right-hand part of Eq. (20) is a complex value even at $\zeta=0$. Purely real eigentunes of similar equations are possible only in exceptional cases.

Therefore Eq. (20) will be investigated further under the assumptions $|\nu| \propto \mu^{2} \ll 1$, that is

$$
Q_{s} \ll \Delta Q_{c} \quad \text { and } \quad\left|\frac{\omega}{\Omega_{0}}-Q_{0}\right| \ll \Delta Q_{c} .
$$

As it was shown in Refs. [6,7], the second inequality follows from the first one if $q=0$. However, now it sets up constraints for the function $q(\tau)$ whose meaning and form should be elucidated.

Thus, we rewrite Eq. (20) in the form

$$
U^{2} \frac{d^{2} \bar{Y}}{d \tau^{2}}=R(\tau)
$$

with the right-hand part

$$
\begin{aligned}
R(\tau)= & \left(\tau+\frac{U^{2}}{\rho} \frac{d \rho}{d \tau}\right) \frac{d \bar{Y}}{d \tau}-\frac{\left(\omega / \Omega_{0}-Q_{0}\right) \Delta Q_{c}}{Q_{s}^{2}} \frac{\rho \bar{Y}}{\rho_{c}} \\
& +\frac{2 \Delta Q_{c} \rho(\tau)}{Q_{s}^{2} \rho_{c}} \int_{\tau}^{1} q\left[\theta_{0}\left(\tau^{\prime}-\tau\right)\right] \bar{Y}\left(\tau^{\prime}\right) \rho\left(\tau^{\prime}\right) \\
& \times \exp (i \varphi) d \tau^{\prime}
\end{aligned}
$$

and

$$
\varphi=\theta_{0}\left(\omega / \Omega_{0}-Q_{0}-\zeta\right)\left(\tau^{\prime}-\tau\right) .
$$

Boundary conditions for Eq. (23) are evident directly from the equation itself through the relations $U^{2}( \pm 1)=0$ which follow from definition (21) and will be reinforced by examples in subsequent sections. Therefore, any appropriate solution of Eq. (22) should satisfy the conditions

$$
R( \pm 1)=0 .
$$

Actually, Eq. (22) will be solved in the paper in two steps. First, the solution will be found at $\varphi=0$ without additional assumptions or approximations like perturbation methods, independently on the wake amplitude. Real eigentunes and TMCI thresholds will be calculated in this stage with high precision. The perturbation technique will be used in the next steps to find the instability growth rate above the TMCI threshold, as well as the chromaticity contribution. A rather serious restriction $\left|\zeta \theta_{0}\right| \lesssim 1$ is actually applied in the last stage. However, it allows one to define at least the sign of the chromaticity required to suppress the instability. More details of the techniques are given below by specific examples.

\section{BOXCAR MODEL, RECTANGULAR WAKE}

A constant wake within a bunch of constant density is considered in this section:

$$
q=q_{0}, \quad \rho(\tau)=\frac{1}{2}, \quad U^{2}=\frac{1-\tau^{2}}{2} \text { at }|\tau| \leq 1 .
$$

This simple model is very pertinent to demonstrate both characteristic features of the phenomenon and the solution procedure to be used.

\section{A. Real spectrum}

First, Eq. (23) has to be solved under assumption $\varphi=0$ which means, in particular, neglect of chromaticity. To satisfy boundary condition (26) in the bunch head, we should take at $\tau=1$ :

$$
\bar{Y}=1, \quad \frac{d \bar{Y}}{d \tau}=\frac{\left(\omega / \Omega_{0}-Q_{0}\right) \Delta Q_{c}}{Q_{s}^{2}} .
$$

With arbitrary trial $\omega$ and these initial conditions, the solution of Eq. (23) can be found step by step to define the function $R(\tau)$ everywhere, including the bunch tail that is the point $\tau=-1$. Applying boundary condition (26) to this point, one can select appropriate values of $\omega$ which are just the bunch eigentunes. In practice, it is a good way to 


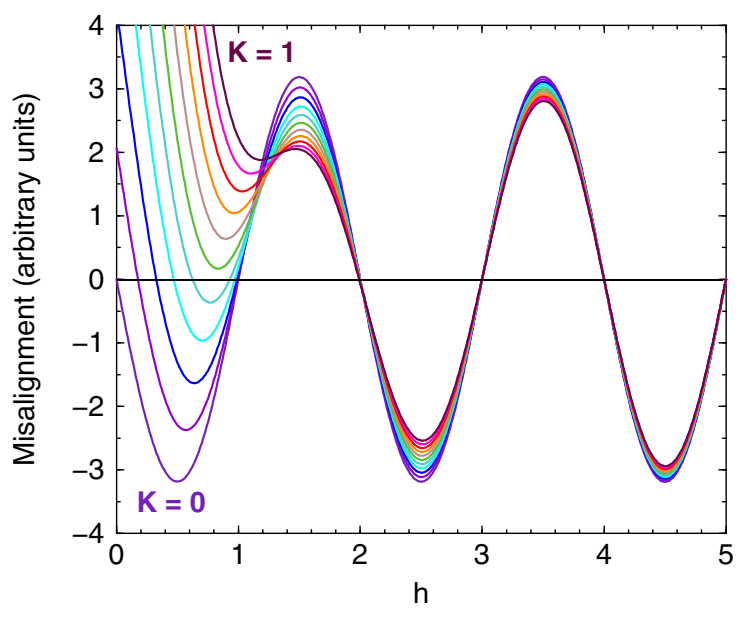

FIG. 1. Misalignment $R(-1)$ vs tune at different positive wakes (boxcar model, rectangular wake).

get real eigentunes including the TMCI thresholds in a designated area. The complex eigentunes above the threshold could be found by this method as well, but it would require essentially more widespread sorting over the complex trial frequencies. Therefore, another technique will be used later to calculate the TMCI growth rate.

The method is illustrated by Fig. 1 where the value $R(-1)$ (misalignment) is plotted against the real frequency at different wakes being presented in terms of the parameters $h$ and $K$ :

$$
h(h+1)=\frac{2\left(\omega / \Omega_{0}-Q_{0}\right) \Delta Q_{c}}{Q_{s}^{2}}, \quad K=\frac{q_{0} \Delta Q_{c}}{Q_{s}^{2}} .
$$

Convenience of such a parametrization is that all the eigennumbers are integers at $K=0: h_{n}=n$. By Fig. 1, most of them almost do not depend on $K$ in the considered interval. There are only two essential exclusions: the eigentunes $h_{0}$ and $h_{1}$ approach each other when the wake increases, and join at $K=0.465$ which is the TMCI threshold. The extended picture is shown in Fig. 2 where several eigentunes are presented in a wider range including negative wakes. It is seen that the second TMCI mode appears at $K \simeq 7.08$ because of merging of the 2 nd and 3rd eigentunes. Next the threshold is found at $K=29$ as a result of coupling of 4th and 5th tunes (not shown in the figure), and other TMCI modes can appear at higher $K$. However, the coupling and the TMCI have never been observed with negative $K$.

\section{B. The TMCI growth rate}

Basic $(K=0)$ eigenfunctions of the boxcar bunch are Legendre polynomials $P_{n}(\tau)$ [3,6]. Eigenfunctions of 0th and 1 st modes are plotted in Fig. 3 at different positive $K$ taken below the first TMCI threshold. They are almost linear functions which can be presented as linear combinations of corresponding basic eigenmodes. With this assumption, the eigenfunctions and eigentunes are

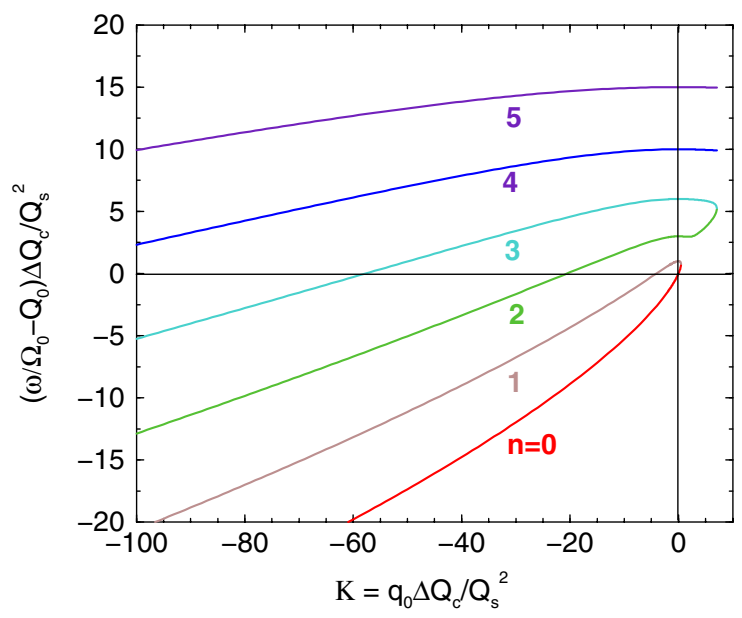

FIG. 2. Eigentunes of several modes vs modified wake strength (boxcar model, rectangular wake).

$$
\bar{Y}(\tau)=1-\frac{2 K \tau}{1-K \mp S} ; \quad \frac{\omega}{\Omega_{0}}-Q_{0}=\frac{Q_{s}^{2}}{2 \Delta Q_{c}}(1+K \pm S),
$$

where

$$
S=\sqrt{1-2 K-\frac{K^{2}}{3}} .
$$

The eigentunes are plotted in Fig. 4 against the parameter $K$. There are two real solutions at $K<0.464$ which are drawn by dashed lines, and two complex conjugated solutions at $K>0.464$ (solid lines). Part of Fig. 2 is also replicated here on a larger scale. Comparison of these curves allows one to conclude that approximate formulas (30) provide a reasonable precision in the range $|\Delta K| \lesssim 2$ around the threshold.

Much different behavior demonstrates a bunch with large negative wake. Examples are given in Fig. 5 where

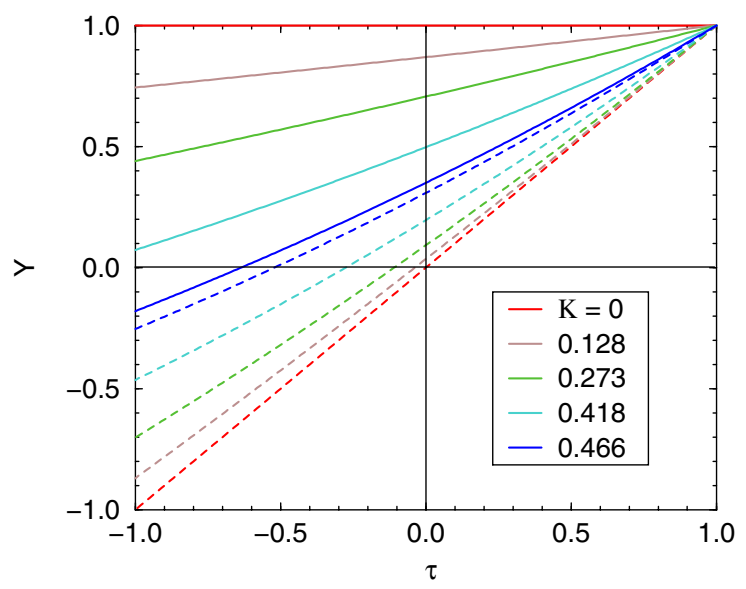

FIG. 3. Eigenfunctions of 0th and 1st modes (solid and dashed lines) at different positive $K$ (boxcar, rectangular wake). 


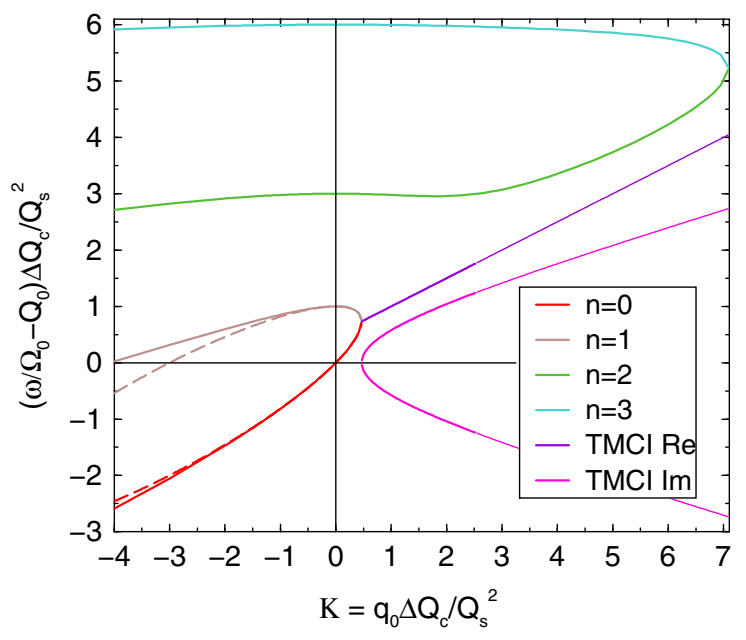

FIG. 4. Eigentunes of lower modes vs the wake strength. Zoom of Fig. 2 + TMCI (boxcar model, rectangular wake).

the lowest eigenfunctions are plotted at several $K<0$. In contrast with Fig. 3, transverse displacement of the bunch accrues approximately exponentially from the bunch head to its tail, and the curves diverge faster if the wake is stronger. Higher modes have similar behavior leading to the conclusion that their junction and the TMCI are impossible at $K<0$. Essential dissimilarity of the eigenfunctions from the basic ones (Legendre polynomials) at $|K| \gg 1$ also deserves attention because this fact restricts applicability of perturbation methods.

\section{Effect of chromaticity}

Considering Eqs. (23) and (24) with $\varphi \neq 0$, we will treat the appearing effect as a small perturbation of the above obtained eigenmodes. This assumption imposes the condition $\left|\zeta \theta_{0}\right|<1$, which may be a rather rigorous restriction, in practice. However, it allows one to determine a sign of chromaticity required to depress the instability, at least.

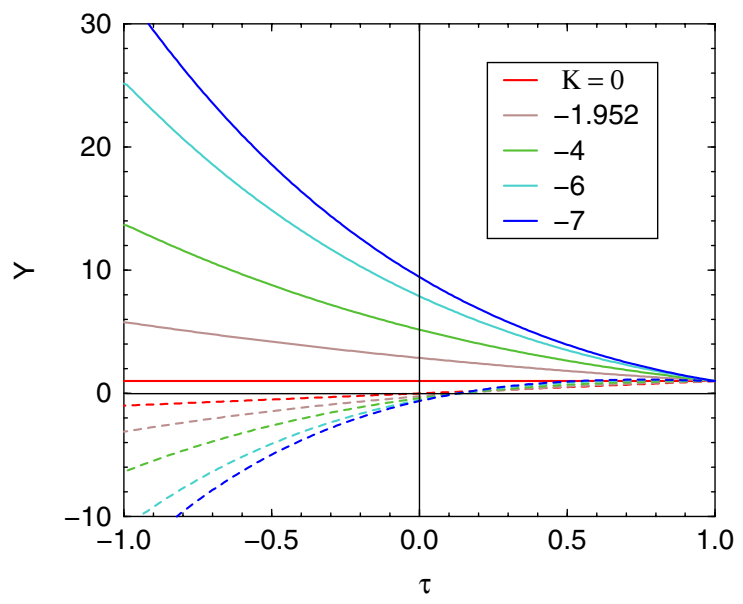

FIG. 5. Eigenfunctions of 0th and 1st modes (solid and dashed lines) with negative $K$ (boxcar model, rectangular wake).

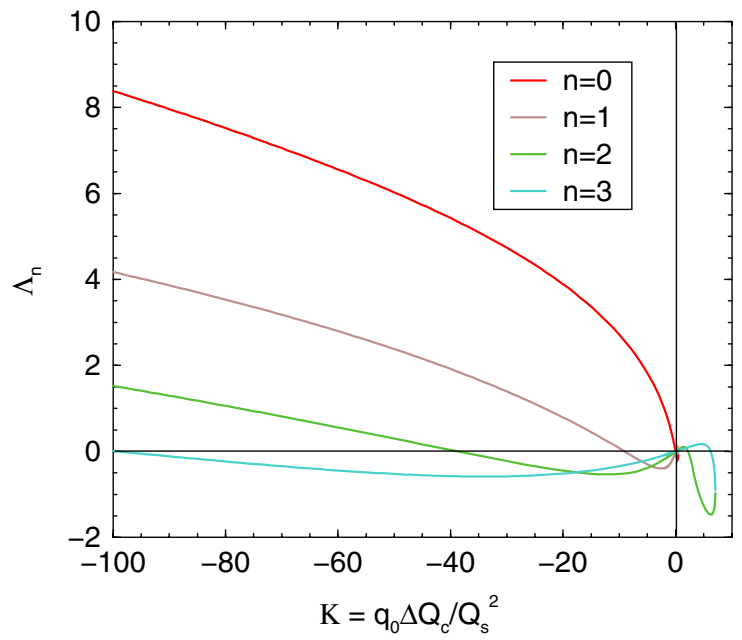

FIG. 6. Chromaticity factors $\Lambda_{n}(B)$ of different modes against the wake strength (boxcar model, rectangular wake).

Imaginary addition to the tune is the most important effect which can be described by the expression:

$$
\operatorname{Im} \frac{\Delta \omega_{n} \Delta Q_{c}}{\Omega_{0} Q_{s}^{2}}=\Lambda_{n}(K) \theta_{0}\left[\zeta-\operatorname{Re}\left(\frac{\omega_{n}}{\Omega_{0}}-Q_{0}\right)\right]
$$

This value is distinct from zero at any chromaticity, both below and above the TMCI threshold. The coefficients $\Lambda_{n}$ are plotted against the wake strength in Figs. 6 and 7 . According to them, chromaticities of opposite signs are needed to depress 0 th or any other mode, when parameter $|K|$ is sufficiently small. Similar behavior is well known in the case of the "usual" (no space charge) head-tail instability $[1,2]$. However, at higher $|K|$, more and more modes can be depressed coincidentally with the 0th one. It is also seen that the sign of chromaticity should be the

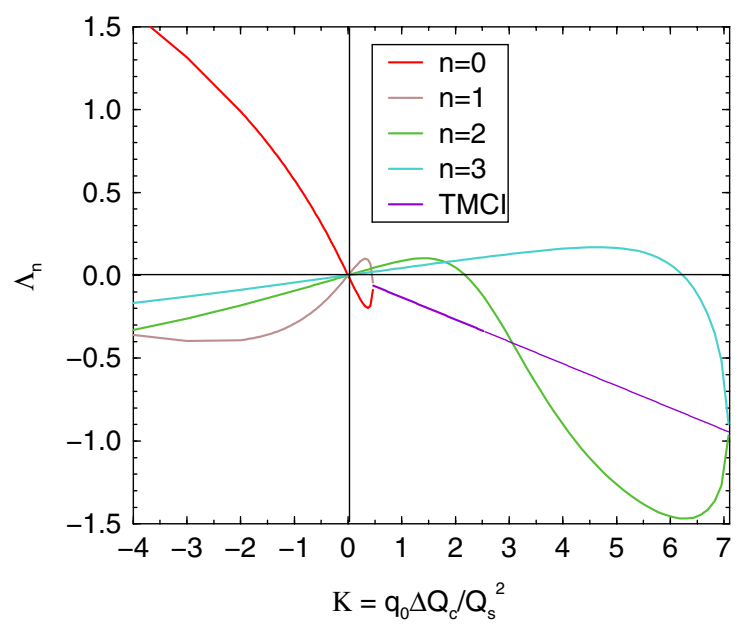

FIG. 7. The same as in Fig. 6, zoom and 1st TMCI mode added (boxcar model, rectangular wake). 
same as the sign of the wake to ensure suppression of the instability.

\section{GAUSSIAN BUNCH, RECTANGULAR WAKE}

Constant wake within a truncated Gaussian bunch is considered in this section:

$$
F \propto \exp \frac{1-A^{2}}{2 \sigma^{2}}-1 \quad \text { at } A \leq 1
$$

Characteristic functions of the bunch involved in Eqs. (23) and (24) are [6]

$\rho(\tau)=C\left[\sigma \sqrt{\frac{\pi}{2}} \exp \left(\frac{1-\tau^{2}}{2 \sigma^{2}}\right) \operatorname{erf}\left(\frac{\sqrt{1-\tau^{2}}}{\sigma \sqrt{2}}\right)-\sqrt{1-\tau^{2}}\right]$

and

$$
U^{2}=\sigma^{2}-C \frac{\left(1-\tau^{2}\right)^{3 / 2}}{3 \rho(\tau)}
$$

where $|\tau| \leq 1$, and $C \simeq \exp \left(-1 / 2 \sigma^{2}\right) /\left(\pi \sigma^{2}\right)$ is a normalizing coefficient. Note that at $|\tau| \simeq 1$

$$
\rho(\tau) \simeq \frac{C\left(1-\tau^{2}\right)^{3 / 2}}{3 \sigma^{2}}\left[1+\frac{1-\tau^{2}}{5 \sigma^{2}}\right], \quad U^{2} \simeq \frac{1-\tau^{2}}{5} .
$$

It was asserted in Ref. [7] that TMCI can arise in a nontruncated Gaussian bunch at zero chromaticity and negative wake corresponding $K<-180$, the instability being provoked by a junction of 2 nd and 3rd base modes. Positive wakes were not presented in the work.

Our calculations lead to different results. The main conclusion is that Gaussian and boxcar bunches are very similar in behavior. In particular, in both cases the TMCI can be caused only by positive wake, has a threshold at $K \simeq 1$, and appears as a confluence of 0 th and 1 st modes, first. Other properties of the mentioned models are very similar also, as it is shown below.

Examples are given for $\sigma=1 / 3$ that is at $3 \sigma$ truncation. Coefficient $C$ is approximately 0.0339 in this case. The solution method is the same as in the previous section, with even simpler initial conditions:

$$
\bar{Y}(1)=1, \quad \bar{Y}^{\prime}(1)=0 .
$$

Dependence of the eigentunes on the wake strength is presented in Figs. 8 and 9 at zero chromaticity. The plots look much like Figs. 2 and 4 which characterize the boxcar model. In particular, the eigentunes do not exhibit a tendency towards coalescence at large negative wakes, and the TMCI is not observed in this region. The statement is sustained by Fig. 10 where several lowest eigenfunctions are plotted. The curves in the graph come apart more and

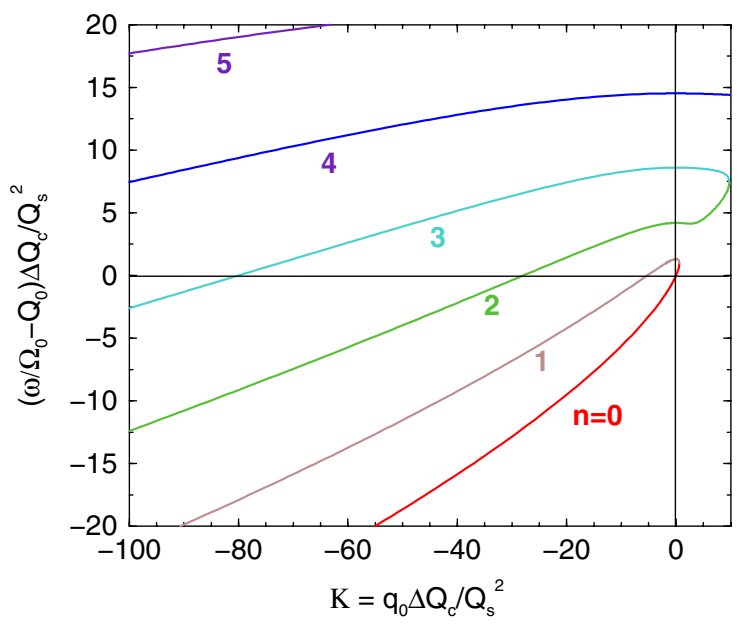

FIG. 8. Eigentunes of several modes against wake strength (Gaussian bunch, rectangular wake).

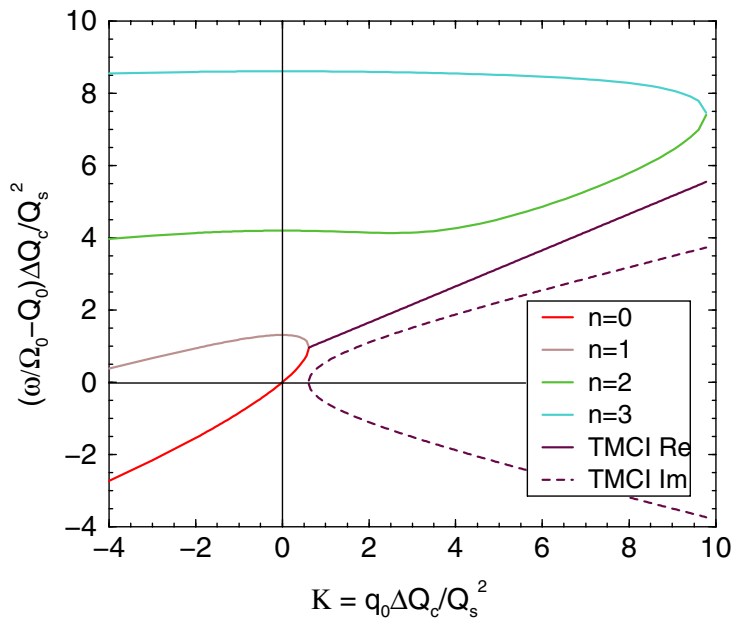

FIG. 9. Eigentunes of lower modes against the wake strength. Zoom of Fig. 8 + TMCI (Gaussian bunch, rectangular wake).

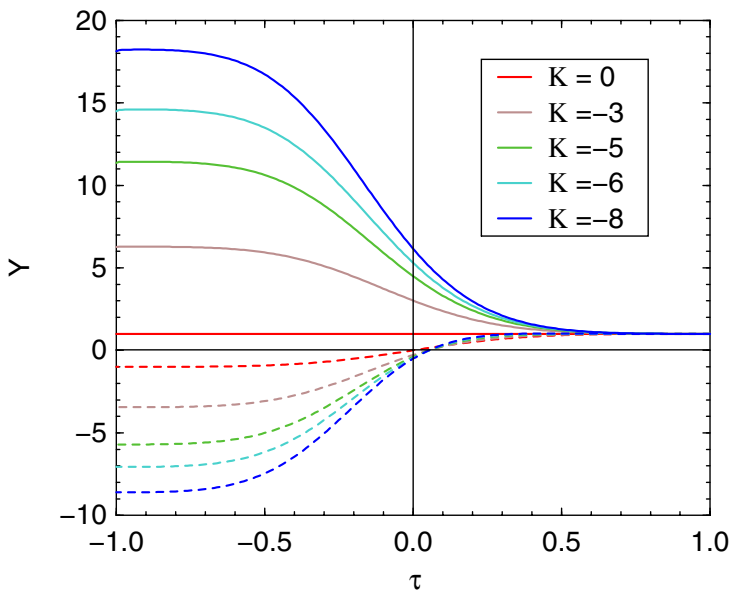

FIG. 10. Eigenfunctions of 0th and 1st modes (solid and dashed lines). Gaussian bunch, negative rectangular wake. 


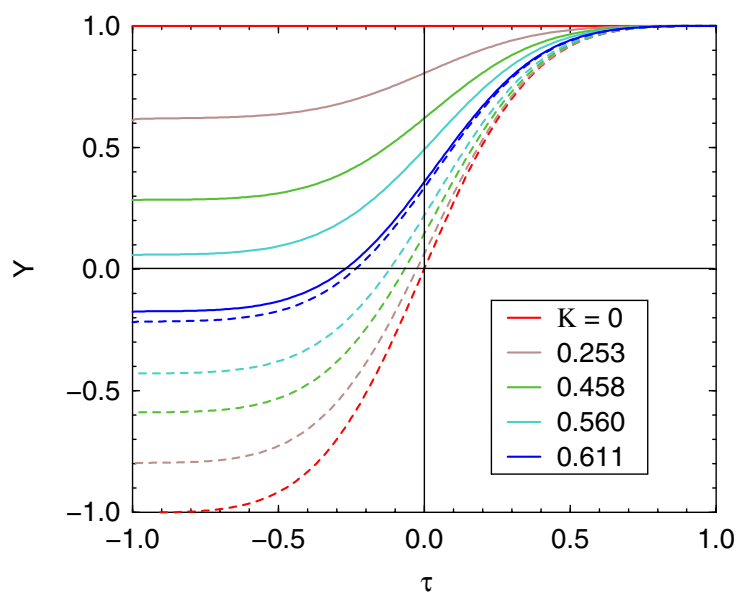

FIG. 11. Eigenfunctions of 0th and 1st modes (solid and dashed lines) before the TMCI appears. Gaussian bunch, positive rectangular wake.

more when the wake is stronger, giving no way to expect the TMCI initiation.

The eigenfunctions of a positive wake have a much different appearance, as it is shown in Fig. 11. A reciprocal approach of 0 th and 1st eigenmodes occurs before the wake parameter reaches threshold value $K=0.612$. Therefore, in the threshold vicinity, the eigenfunctions are adequately representable as a linear combination of corresponding basic eigenfunctions (red lines in Fig. 11). Other basic modes give an unessential contribution because their tunes differ by at least several units. The TMCI growth rate can be obtained by applying this assumption above the threshold as it is shown in Fig. 9. The second TMCI threshold appears at $K=9.78$ because of coalescence of $2 \mathrm{nd}$ and $3 \mathrm{rd}$ eigenmodes. Chromaticity effects are described by Eq. (32) with the coefficients $\Lambda_{n}$

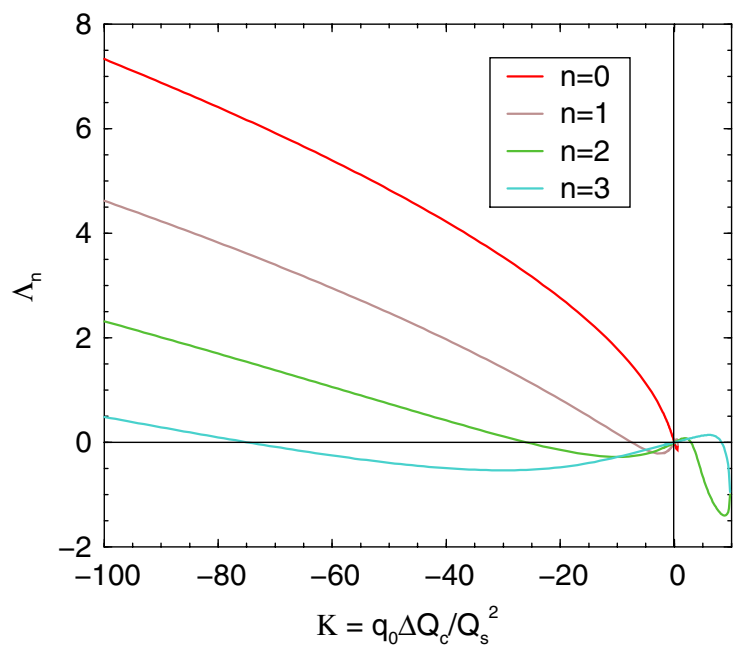

FIG. 12. Chromaticity factor $\Lambda_{n}(K)$ for several modes against the wake strength (Gaussian bunch, rectangular wake).

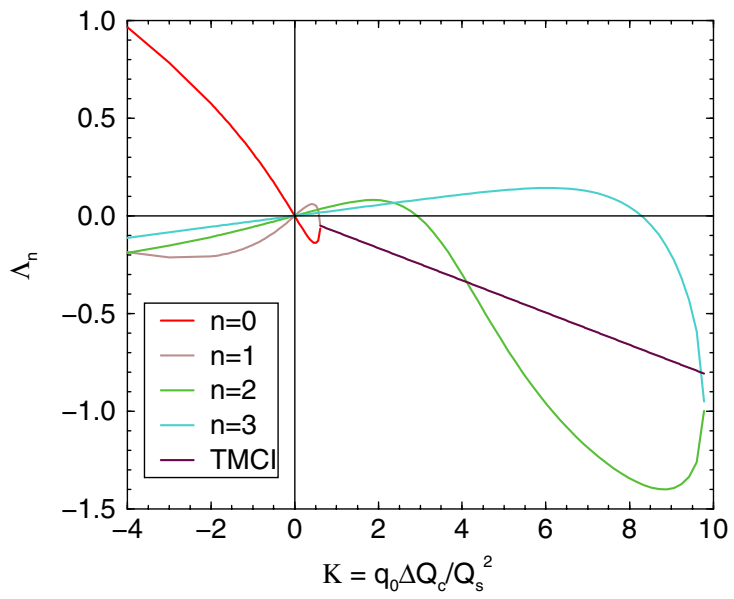

FIG. 13. Zoom of Fig. 12 to show two TMCI thresholds (Gaussian bunch, rectangular wake). Chromaticity factor above first TMCI threshold is shown as well.

which are shown in Figs. 12 and 13 being very similar to Figs. 6 and 7.

\section{GAUSSIAN BUNCH, EXPONENTIAL WAKE}

The Gaussian bunch presented in the previous section is combined in this section with the exponential wake:

$$
W(t) \propto \exp \left(-\frac{t}{t_{0}}\right)
$$

The same solution procedure is adaptable and effective in this case because the wakefield factor of Eq. (24) breaks up into independent parts:

$$
q\left[\theta_{0}\left(\tau^{\prime}-\tau\right)\right]=q_{0} \exp (\alpha \tau) \exp \left(-\alpha \tau^{\prime}\right)
$$

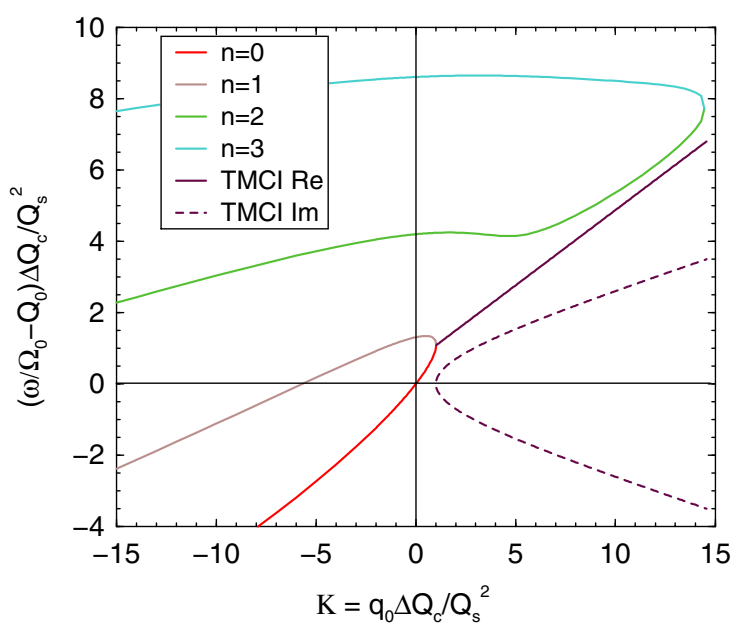

FIG. 14. Eigentunes of lower modes including TMCI with positive wake (Gaussian bunch, exponential wake). 


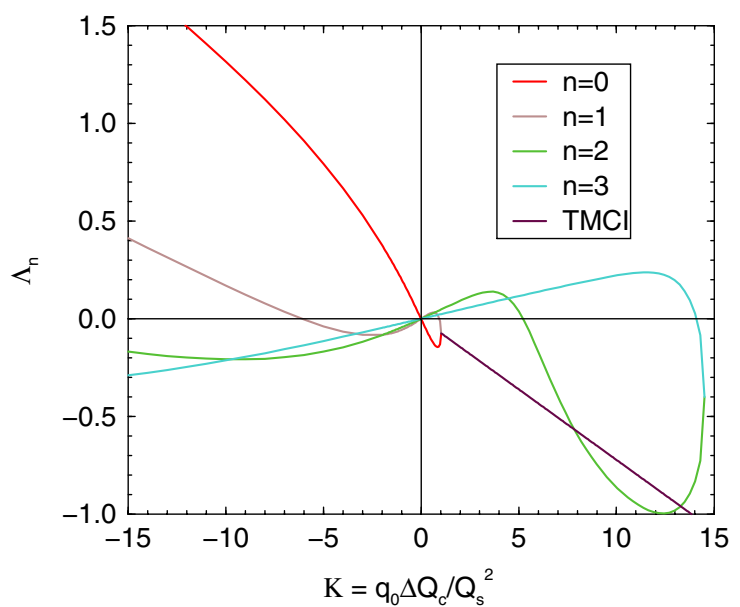

FIG. 15. The same as in Fig. 6 at positive wake. Two TMCI thresholds are seen (Gaussian bunch, exponential wake).

The results are presented in Figs. 14 and 15 at $\alpha=1$ being very similar to the case of rectangular wake. As before, TMCI is possible only with positive wake, although the TMCI thresholds are a bit higher: 1.03 instead of 0.612 and 14.4 instead of 9.78 (Fig. 14). The depression factor $1.5-1.7$ is quite an expected result of averaging of the decaying wake over the bunch. Chromaticity effects are attenuated by the same factor (Fig. 15).

\section{CONDITIONS OF APPLICABILITY}

The basic assumptions of the paper are brought in Eq. (22) and include the bunch eigentunes which are unknown a priori. Of course, a wide diversity of the spectra is possible depending on the wake form. However, presented results allow one to state the conditions at least for monotonic wakes.

It follows from Figs. 2, 8, and 14 that, on a global scale, the lower tunes are about proportional to the function $q(\tau)$ averaged over the bunch length, being 2-3 times less in value. This allows one to rewrite Eq. (22) in the form

$$
Q_{s} \ll \Delta Q_{c}, \quad|\bar{q}| \ll \Delta Q_{c} .
$$

It means that the synchrotron tune is small, and the space charge dominates in the impedance budget far exceeding the wake contribution. In spite of this, the wake can cause a radical change of the bunch spectrum as it was shown above. This phenomenon is explained by a significant degeneration of the basic bunch spectrum at low synchrotron frequency, when a lot of different eigenfunctions have very close eigentunes. This fact was established in Refs. [3,6,7] and is confirmed in this paper. Its extreme manifestation is that, at $Q_{s}=0$ and with no wake, any function $Y(\theta)$ satisfies Eq. (11) with the eigenfrequency $\omega=\Omega_{0} Q_{0}$.
The mentioned circumstance essentially restricts applicability of the perturbation methods of the solution. As it follows from Fig. 4, similar methods are suitable at the additional condition

$$
|K|=\frac{|q| \Delta Q_{c}}{Q_{s}^{2}} \lesssim 1
$$

but a special analysis is required if it is violated.

\section{CONCLUSION}

Transverse instability of a bunch is studied with synchrotron oscillations, space charge, and wakefield taken into account. Most of the presented results are obtained with no use of perturbation methods. In particular, it concerns the bunch spectrum including the transverse mode coupling instability thresholds. It is shown that, with zero chromaticity and monotonically decreasing wakes, only positive wakes can excite the TMCI by a coupling of neighboring eigenmodes. The lowest TMCI threshold is located at $K \simeq 1$ for any bunch shape. Therefore the perturbation theory is usable to determine the instability growth rate only near the threshold. Effects of chromaticity are studied with the additional assumption that contributed phase advance is $\lesssim 1$ within the bunch.

Doubtless, the resistive wall wake $q \propto-1 / \sqrt{\tau}$ is one of the most interest cases, in practice. Such a wake decays rather slowly so that multibunch and multiturn effects should be necessarily taken into account for a full description of the phenomenon. This specific problem has to be studied separately. Nevertheless, one can expect that this negative wake does not cause a confluence or approach of the eigenmodes and cannot produce TMCI-like effects.

\section{ACKNOWLEDGMENTS}

FNAL is operated by Fermi Research Alliance, LLC under Contract No. DE-AC02-07CH11395 with the United States Department of Energy.

[1] C. Pellegrini, Nuovo Cimento A 64, 447 (1969).

[2] M. Sands, Report No. SLAC TN-69-8, 1969.

[3] F. Sacherer, Report No. CERN-SI-BR-72-5, 1972.

[4] V. Balbekov, Zh. Tekh. Fiz. 46, 1470 (1976) [Sov. Phys. Tech. Phys. 21, 837 (1976)].

[5] M. Blaskiewicz, Phys. Rev. ST Accel. Beams 1, 044201 (1998).

[6] V. Balbekov, Phys. Rev. ST Accel. Beams 12, 124402 (2009).

[7] A. Burov, Phys. Rev. ST Accel. Beams 12, 044202 (2009); 12, 109901 (2009).

[8] D. Mohl and H. Shonauer, in Proceedings of the IX International Conference on High Energy Accelerators, Stanford, 1974 (AEC, Washington, D.C., 1974), p. 380. 